\title{
From current trace to the understanding of confined media
}

\author{
Jean Roman ${ }^{1}$, Bruno Le Pioufle ${ }^{2}$, Loïc Auvray ${ }^{3}$, Juan Pelta ${ }^{1}$, and Laurent Bacri ${ }^{1 \mathrm{a}}$ \\ 1 LAMBE, Univ Evry, CNRS, CEA, Université Paris-Saclay, F-91025, Evry, France \\ 2 ENS Paris-Saclay, CNRS, SATIE, UMR 8029, Cachan, France \\ 3 Matière et Systèmes Complexes, Université Paris Diderot/CNRS (UMR 7057), 75205, Paris, Cedex 13, France.
}

Received: date / Revised version: date

\begin{abstract}
Nanopores constitute devices for the sensing of nano-objects such as ions, polymer chains, proteins or nanoparticles. We describe what information we can extract from the current trace. We consider the entrance of polydisperse chains into the nanopore, which leads to a conductance drop. We describe the detection of these current blockades according to their shape. Finally, we explain how data analysis can be used to enhance our understanding of physical processes in confined media.
\end{abstract}

PACS. 71.20.Rv Polymer condensed matter - 82.37.-j Single-molecule kinetics

\section{Introduction}

At the cell scale, many ions and biomolecules are transported through channels in the cell membrane. These channels consist of membrane proteins. Each of them has their own insertion mechanism into the cell membrane. [1-3] While the study of ion channels is common in electrophysiology, confinement phenomena involved in these nanometric holes are less understood in soft matter.

Since the seventies, economic interest in oil extraction required the understanding of physical or chemical processes involved during the drilling of oil wells. One current technique uses drilling muds to increase the well production. These fluids interact with the reservoir. They lead to instability birth in porous media, similar to the viscous fingers observed in a Hele-Shaw cell. $[4,5]$ The viscosity of these muds was controlled by addition of additives, such as polymers. In this context, understanding the interactions between these polymer chains and limestone or sandstone rocks was a source of scientific questioning on the confinement in porous media and gave rise to numerous theoretical works by P. G. De Gennes or F. Brochard-Wyart. [6-10] These results could be extended to size exclusion chromatography, [11] capillary electrophoresis [12] or DNA transfection. [13,14]

Despite these numerous theoretical works, their experimental validation is rare and requires neutron scattering techniques. [15-17] In this context, L. Auvray was really interested by Kasianowicz's and colleagues' work. [18] The latter showed that DNA translocation through a protein channel could be observed by electrical measurements at the single molecule scale. The great benefit from

\footnotetext{
Send offprint requests to:

a Present address: laurent.bacri@univ-evry.fr
}

this method is to be able to observe the conformation of each macromolecule in the nanopore such as polyelectrolytes, [19-21] DNA, [18,22] polysaccharides, [23] proteins, [24-26] nanoparticles. [27-29]

When one of these analytes is in the vicinity of the pore or enters into it, the ionic conductance suddenly decreases and such events are referred to as blockades. Their detection is of interest. Following an approach initiated by Loïc Auvray, in this publication, we describe some algorithms used to detect and characterize current blockades according to their shape (short, long, one or multi-step). Then, we illustrate our discussion from the trace obtained with poly(ethylene glycol) chains in presence of an Aerolysin channel from Aeromonas hydrophila. [30-32]

From the distribution of blockade frequency, duration or amplitude, we perform a statistic data analysis to calculate the characteristic frequency or dwell time. These parameters lead to the knowledge of the magnitude of the energy barrier, which governs the confinement and the transport into the nanopore. [33-35]

\section{Experimental section}

\subsection{Chemicals and solutions}

$\mathrm{KCl}$ and decane are bought from Sigma-Aldrich. Diphytanoylphosphocholine (DPhPC) lipids are furnished by Avanti Polar Lipids Inc. Electrical signals are measured by an Axon Patch 200 B (Molecular devices) amplifier. Data are acquired with a PCI-6251 National Instruments card, managed by an homemade software. Data are processed according to functions written with Igor Pro software (Wavemetrics Inc). Aerolysin is synthesized following the 
method described in [36] by Manuela Pastoriza-Gallego. [25]

\subsection{Channel insertion}

We use a device previously described. [37-39]. Briefly, we consider two compartments separated by an insulating lipid bilayer and filled with an electrolyte solution, such as $\mathrm{KCl}$ buffer. The bilayer is prepared from a $1 \%$ of DPhPC lipids diluted in decane and is spread over a pinhole. The potential difference is applied with two $\mathrm{Ag} / \mathrm{AgCl}$ electrodes. The transport of PEG 1500 chains into Aerolysin channel are performed by using $3 \mathrm{M} \mathrm{KCl}$ buffered with 5 mM HEPES, $\mathrm{pH}=7.5$ (fig. 1(a)).

\section{Results and discussion}

\subsection{Blockade detection}

The reliability of blockades detection is crucial in the nanopore technique, which has known a strong development for twenty years. $[18,40]$ We can find some commercial (Clampfit, Molecular Device) or free softwares (WinEDR, University of Strathclyde), but they are mainly dedicated to electrophysiology applications. Currently, in the nanopore community, each team designs its own solution developed by using data analysis software (Matlab, Igor Pro, Origins), while using mainly the same algorithms. For some years, several publications have especially been dedicated to data analysis, [26,41-45] which was previously shortly described in the "Set up and Material" part of publications or in their supplementary data. [46]. Shortly, data analysis contains three steps: 1) blockade detection, 2) blockade relevancy, 3) blockade characterization. After this step, we shall describe how we plot data distribution to extract an understanding of the physical processes involved in confined media.

Ionic current traces are usually acquired with a fixed sampling rate $f_{\text {sam }}$. This frequency is currently about 250 $\mathrm{kHz}$, [22] [25] but can go up to $4.19 \mathrm{MHz}$. [48] For this work, we consider just the usual value $f_{\text {sam }}=250 \mathrm{kHz}$, allowing a sampling time of $4 \mu \mathrm{s}$. When we measure the ionic current trough the nanopore, we observe a noisy baseline characterized by its average value $I_{0}$ and its standard deviation $\sigma$. Considering that the blockade amplitude exceeds the noise level, we reject all the fluctuations, which amplitudes are smaller than an arbitrary threshold $k \sigma$, where $k$ is a positive constant (fig. 1(b)). We can arbitrary choose $k=2$ to reject $95.5 \%$ of the signal, [19,42], or $k=6$ to reject more than $99.9 \%$ of the signal. [41] If this threshold criterion is too large, we can miss some blockades with small amplitudes. In the case of baseline fluctuations, this method is applied on multiple small windows inside the waveform. [41,44] While Pedone et al. use a small window (typically 100 data points), Plesa et al. use a larger one (typically 1000 data points). Usually, the Bessel low-pass filter cut-off frequency is about $10 \mathrm{kHz}$, i.e. a $100 \mu \mathrm{s}$ critical event duration. Considering a)

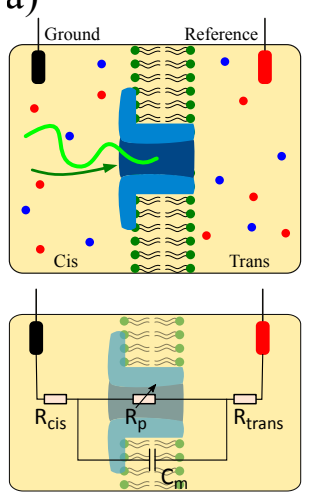

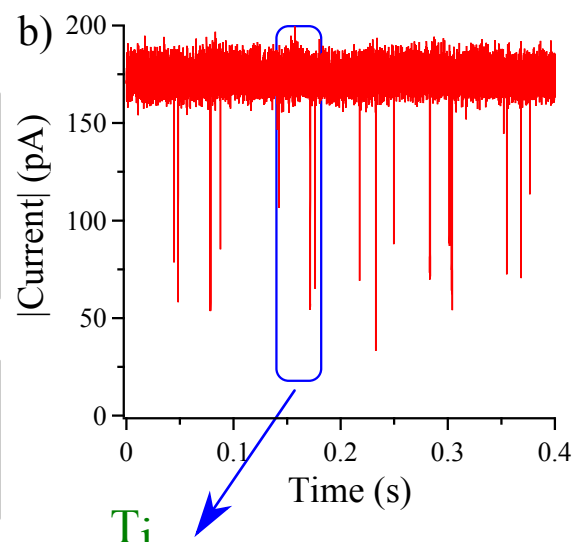

c)

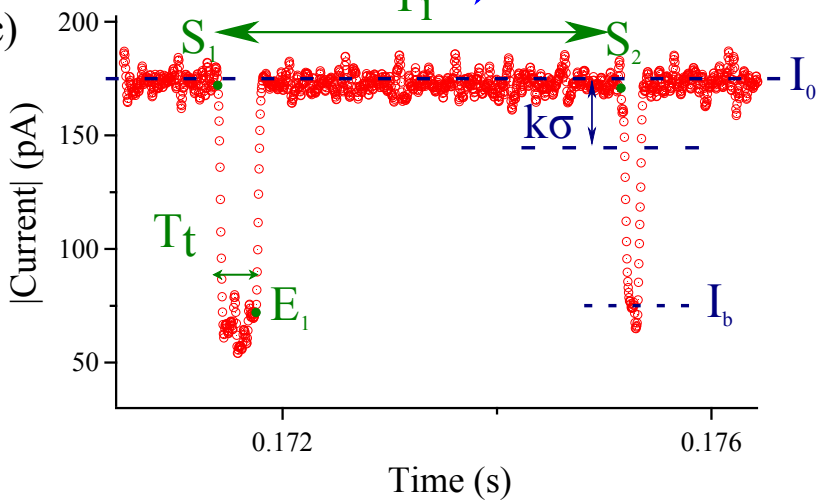

Fig. 1. Macromolecule transport through a single Aerolysin channel. (a) (above) Electrical detection of a poly(ethylene glycol) chain (PEG 1500) through an Aerolysin channel. (below) Electrical circuit model of the channel inserted in a membrane. $R_{p}$ and $C_{m}$ are the channel resistor and the membrane capacitor respectively. $R_{\text {cis }}$ and $R_{\text {trans }}$ are the access resistance on the cis and trans side respectively. [47] (b) Trace of the ionic current through this channel of a $10 \mu \mathrm{M}$ PEG1500 solution in a $3 \mathrm{M} \mathrm{KCl}$ buffered with $5 \mathrm{mM}$ HEPES at $\mathrm{pH}=7.5$, under a $-120 \mathrm{mV}$ voltage. (c) Focus on two blockades. The dwell time $T_{t}$ is defined as the duration between the Start point $\left(S_{1}\right)$ and the End Point $\left(E_{1}\right)$. The inter-event time $T_{i}$ is the duration between two successive blockades $\left(S_{2}\right.$ and $\left.S_{1}\right) . I_{0}$ and $I_{b}$ correspond to the base current and the blockade one respectively . $k \sigma$ is the magnitude of the detection threshold.

the above-mentioned sampling rate, this period is corresponding to 25 data points. Then, the Pedone's criterion calculates the average and standard deviation $\sigma$ by using four periods and the Plesa's one uses 40 periods. Nevertheless, if the blockade frequency is higher than 250 $\mathrm{Hz}$, the moving average is slightly modified by the presence of these blockades. That's why Plesa et al. developed a clever recursive algorithm combining average calculation and blockade detection. [44] Another method is to directly erase the noise component from the trace with a non-linear filter. The CUSUM method is an efficient algorithm allowing to transform a raw trace into a succession of line segments. [49] The remaining fluctuations are corresponding to the blockades, provided the good choice of just one filtering parameter. 


\subsection{Blockade relevancy}

After the blockade detection step, we observe that the latter could have several origins 1) electrical noise, 2) the "attacks" due to the interactions between the nanopore and the macromolecules, which do not succeed in overcoming the entrance energy barrier [25] 3) the macromolecule transport through the nanopore. The blockades of type 1) could be removed by using a higher amplitude threshold: $3 \sigma,[19] 5 \sigma,[49,50] 6 \sigma$. [41] Nevertheless, "attacks" and "transport" through the nanopore could not be discriminated by using just amplitude threshold. This discrimination step could be performed by taking both blockade amplitudes and durations into account (fig. 4). We can note an interesting method proposed by A. Radenovic and colleagues, who use a duration threshold to discriminate between relevant blockades and short pulses. [49]

Finally, we can cite an interesting algorithm developed by J. Behrends and colleagues, which is described in detail in the supporting information of ref. [46]. Here, the threshold is not based on the current amplitude, but on its variation i.e. the current derivative. This method was developed to quickly detect the negative or positive transitions. Considering that the baseline fluctuations are relatively smooth, while the blockade transitions are sharper, the discrimination is performed with a variation magnitude threshold: all the variations between two successive points smaller than $15 \mathrm{pA}$ (or $3 \mathrm{pA} / \mu$ s according to the sampling rate) are rejected. [46] The advantage of this algorithm is that we don't need to define a moving window to detect the blockades if the baseline fluctuates. Now, we shall see what kind of information we can extract from this blockade discrimination.

\subsection{Blockade characterization}

Each blockade is defined by its duration $T_{t}$ and amplitude $\Delta I$. It begins when the current is equal to the average one $I_{0}$ calculated in the moving window just before the blockade (Start point, $S_{1}$ in fig. 1 (c)). It ends at the end of the plateau or at the minimum position of the blockade (End point, $E_{1}$ in fig. 1 (c)). [41] Another parameter to take into account is the inter-blockade time $T_{i}$ between two successive blockades (start points $S_{1}$ and $S_{2}$ in fig. 1(c)).

This blockade sensing could be enhanced by a secondorder-differential-based calibration (DBC) method, which is based on the determination of the inflection points of the blockades. [43] To avoid the influence of the noise, this calculation uses a Fourier series fit of each blockade. Another point of view is to consider that the blockade duration is equal to the Full Width at Half Maximum (FWHM). [44] Finally, both points of view lead to the same result. [41] This approach is available for most of the blockades, but sometimes we have to improve it to take short or multi-level blockades into account (fig. 2).
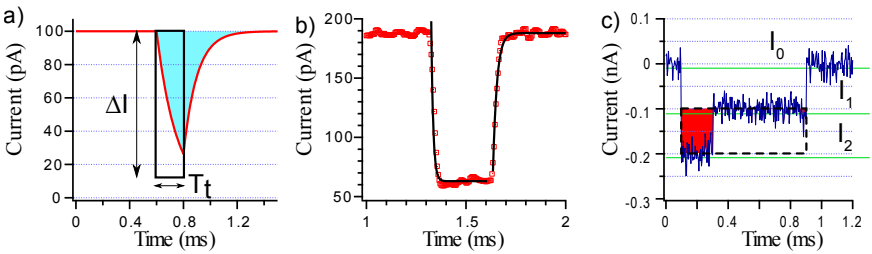

Fig. 2. Short blockades and multi-level analysis. (a) Poly(DA)60 through a $\alpha$-Hemolysin channel. Measure of the blockade current $\Delta I$ by an integration method. The blue integrated area of the trace corresponds to the Event Charge Deficit (ECD) and is equal to the box area of duration $T_{T}$ and amplitude $\Delta \mathrm{I}$. $1 \mathrm{M}$ KCl. $\Delta \mathrm{V}=100 \mathrm{mV}$ (adapted from [43]) (b) PEG 1500 through an Aerolysin channel. The current blockade is fitted by two exponential functions (adapted from [42]). 3M KCl. $\Delta V=-100 \mathrm{mV}$. (c) Detection of DNA blockade through a solid-state nanopore. The first current level $I_{1}$ corresponds to a single DNA inside the nanopore, the second one $I_{2}$ when two DNA chains enter the nanopore. The integrated area of the trace between these both levels (ECD) is painted in red. $I_{0}$ is the local base level of the trace. $1 \mathrm{M} \mathrm{KCl}$ (adapted from [44]).

\subsection{Short blockades}

Now, we can focus on the short blockades. We suppose that the signal is filtered before the acquisition. Considering a low-pass filter characterized by its cut-off frequency $f_{c}$, the bandwidth of this filter (i.e. $f_{c}$ ) and rise time $T_{r}$ are related according to the relation : $f_{c} \times T_{r}=0.33$ for a fourth-order low-pass Bessel filter. [43] Then, blockades shorter than $2 \times T_{r}=0.66 / f_{c}$ are distorted by the filter. Thus, some methods were developed to overcome this limitation.

A first one is to calculate the integrated area of the blockade, which is also equal to the Event Charge Deficit $(E C D,[43,44])$ and reads $\mathrm{ECD}=\int\left(\mathrm{I}_{0}-\mathrm{i}(\mathrm{t})\right) \mathrm{dt}$ (fig. 2(a)).

During a blockade, the charge $q_{N}$ inside the nanopore reads $q_{N}=\Delta I \times T_{t}$, where $\Delta I$ is the unmodified current amplitude of the blockade. [43] As the channel is electrically modeled with a capacitor in parallel with a resistor, this charge leads to the load and unload of the capacitor leading to a signal distortion. As there is charge conservation between the beginning and the end of the blockade, the ECD is equal to $q_{N}$. The advantage of this method is that it is easy to use. Nevertheless, it is based on the assumption that the blockade duration remains constant despite the low-pass filter. The uncertainty increases when the blockade duration is reduced by the low-pass filter. Nevertheless, this ECD method allows to obtain an apparent cutoff frequency two times greater than that of the conventional method, which is a great improvement. [43]

A second method was implemented by Balijepalli and colleagues in the ADEPT algorithm. $[42,51]$ It is based on the assumption that the channel and membrane are described as an electrical circuit analog where the channel resistance increases when a polymer chain enters the pore (fig. 1 (a)). [42] Then, the Laplace transformed current $I(s)=V / Z(s)$, where $Z(s)$ is the circuit impedance, reads : 


$$
I(s)=\frac{a(s+b)}{s(s+1 / \tau)}
$$

where $a=V /\left(R_{\text {cis }}+R_{\text {trans }}\right), b=1 /\left(R_{p} C_{m}\right)$, and $\tau=R_{p} C_{m}\left(R_{\text {cis }}+R_{\text {trans }}\right) /\left(R_{p}+R_{\text {cis }}+R_{\text {trans }}\right)$. [42] The inverse Laplace transform leads to an exponential decay for $t>0$ :

$$
i(t)=\alpha \exp (-t / \tau)+i_{0}
$$

where $\alpha=a(1-b \tau)$ and $i_{0}=a b \tau$ is the empty channel current. Then, this relation could be generalized by considering $N$ conductance states :

$$
I(t)=I_{0}+\sum_{j=1}^{N} a_{j}\left(1-\exp \left(-\frac{t-\mu_{j}}{\tau_{j}}\right)\right) H\left(t-\mu_{j}\right)
$$

where $a_{j}$ the amplitude of the $j^{t h}$ step, $\tau_{j}$ the time constant of the system, $\mu_{j}$ the start point of the step and $H(t)$ the Heaviside step function. Following this model, a blockade is described by two steps $(N=2)$. In the case of short durations, this model returns the "true shape" of the blockade ( $T_{t}$ and $\left.\Delta I\right)$ (fig. 2(b)). In a context of fast DNA sequencing, and current traces containing complex multilevel blockades, this model allows the determination of each discrete step $(N=26)$. [42] The advantage of this method is that it is adapted to long and short-lived events. In the case of short-lived events, the sampling frequency must be increased ( $\mathrm{fs}=500 \mathrm{kHz}$ instead of $200-250 \mathrm{kHz}$ ) to reduce the uncertainty on the fitting parameters.

Long and his colleagues have developed a new algorithm to avoid the data filtering and signal distortion. They successfully apply a Modified Hidden Markov Model (MHMM) to a raw signal, characterized by low current blockades and strong signal-noise rate $(\mathrm{SNR} \approx 3)$. [45] This method at least doubles the number of detected events with duration shorter than $2 \times T_{r}$ (see above) compared to previous methods. [41,43] Recently, the same team has developed an analysis method based on the Hilbert-Huang Transform to study single analytes through the confined space of an Aerolysin channel. [52]

Another type of complex blockades analysis is the discrimination between two situations: a single or two macromolecules enter the pore together (fig. 2(c)). In the first case, the current trace is composed by one step at the current level $\left(I_{1}\right)$. In the second case, the trace is described by two steps : the first one at the level $\left(I_{1}\right)$ due to the dwelling of a single chain, the second one at the level $\left(I_{2}\right)$ due to the presence of two chains inside the nanopore at the same time. [44] The discrimination between both these blockade types is performed by the calculation of the area of the current trace between theses levels $I_{1}$ and $I_{2}$. Drawing the distribution of these areas allows the determination of the area threshold between both local structures of the macromolecule. The blockade detection leads to a great amount of data, which must be statistically processed to broach the physical processes involved in confined media.

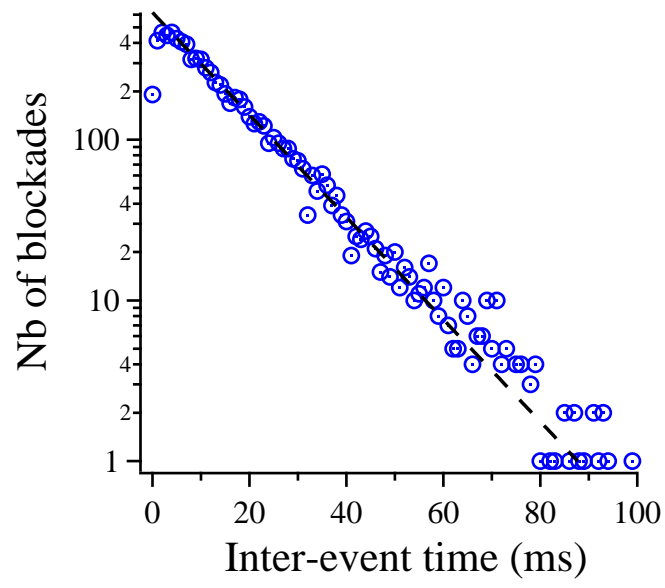

Fig. 3. Macromolecule entrance into a confined channel. Distribution of the time $T_{i}$ between two following blockades. The distribution is fitted with an exponential function $\exp \left(-f \times T_{i}\right)$. Aerolysin channel in presence of $10 \mu \mathrm{M}$ PEG1500 solution in a 3 $\mathrm{M} \mathrm{KCl}$ buffer at $-120 \mathrm{mV}$.

\subsection{Physical processes in confined media}

\subsubsection{Polymer chain confinement}

The inter-blockade time $T_{i}$ is related to interactions between macromolecules and the entrance of the nanopore. As there is no correlation between two following current blockades, this distribution follows a Poisson statistic. This one decreases according to an exponential law $\exp \left(-f_{c} T_{i}\right)$, where $f_{c}$ is the characteristic blockade rate (fig. 3). When poly(ethylene glycol) chains (10 $\mu \mathrm{M}, \mathrm{PEG}$ 1500 in $3 \mathrm{M} \mathrm{KCl}, \Delta \mathrm{V}=-120 \mathrm{mV}$ ) is in presence of an Aerolysin channel, we measure an average frequency of $f_{c}=73.3 \pm 0.8$ blockades/s (fig. 3). Now, we can evaluate the magnitude of this frequency from the Fick's law. $[20,25,53,54]$ Briefly, the diffusion flux vector $\boldsymbol{J}$ is proportional to the concentration gradient according to the relation : $\boldsymbol{J}=-D \operatorname{grad}(c)$ where $D$ is the diffusion coefficient, $c$ the PEG concentration. At first glance, we could assume that this gradient is constant, then we write $\operatorname{grad}(\mathrm{c})=-\mathrm{C} / \ell$ where $C$ is the bulk PEG concentration in the cis compartment and $\ell$ the channel length. In this calculation, we neglect the concentration in the trans side compare to the one in the cis side. Then, $J=D C / \ell$. Finally, the number of particles entering the channel per second is given by : $\nu=J \times \mathcal{A}$ where $\mathcal{A}$ is the internal section area of the Aerolysin. Finally, we write the relation :

$$
\nu=C D \mathcal{A} / \ell
$$

$D$ is evaluated from the Stokes-Einstein equation : $D=\frac{k_{B} T}{6 \pi \eta R_{g}}$ where. $R_{g}=a / \sqrt{6} N^{0.5}$ is the gyration radius of the PEG chain, where $a$ is the length of the monomeric unit and $N$ the number of these units. If $C=$ $10 \mathrm{mM}, D=310^{-10} \mathrm{~m}^{2} . \mathrm{s}^{-1}, \mathcal{A}=810^{-19} \mathrm{~m}^{2}$ and $\ell=$ 
$10 \mathrm{~nm}[30,32]$, then $\nu=14010^{3}$ blockades/s. This frequency is higher than the one we measure, because we must take the confinement into account. During this process, the polymer chain must overcome an energy barrier to enter the channel. Then, the frequency reads for neutral chains :

$$
f_{c}=\nu \exp \left(-U^{\star} / k_{B} T\right)
$$

where $U^{\star} \approx 7.6 k_{B} T$ is the confinement energy or barrier height. This amplitude is larger than the one measured with unfolded protein MalEwt in $1 \mathrm{M} \mathrm{KCl}$ and $1.5 \mathrm{M}$ guanidium $\left(\approx 4 k_{B} T\right)$ [25] or the one with pertactin in $1 \mathrm{M}$ $\mathrm{KCl}$ and $1 \mathrm{M}$ guanidium $\left(\approx 2.5 k_{B} T\right)$ [54]. Nevertheless, it is similar to the one measured with PEG 2000 in $2 \mathrm{M} \mathrm{KCl}$ in presence of an $\alpha$-Hemolysin channel $\left(6.41 k_{B} T\right)$. [55] In this situation, PEG chains can not be considered as neutral chains and are slightly positively charged, due to their affinity with $\mathrm{K}^{+}$ions. [56] Then, the magnitude of this barrier could be increased by electrostatic repulsion between the confined chain at the entrance of the nanopore. Moreover, the electro-osmosis flow is opposed to the electric force, which is the driving force for charged chains. [57] This equilibrium between these both processes modifies the height of the energy barrier $U^{\star}$, which is lowered by the electrostatic energy $U_{e}=z \mathrm{e} \Delta \mathrm{V} / k_{B} T$ where $z$ is the apparent number of elementary charges e of the PEG chain. $[25,53,55]$

\subsubsection{Polymer chain Transport}

Now, we perform a scatter plot of the current blockade $I_{b}$ as a function of dwell time $T_{t}$. This graphic representation allows to have a look at transport phenomena inside the nanopore (fig. 4 (b)). Nevertheless, the physical parameters are measured from the statistical distribution of $I_{b}$ and $T_{t}$.

Considering the first parameter, the blockade current histogram does not lead to a Gaussian distribution (fig. 4(a)), but shows several peaks. Each of them could be associated to the chain length in the polydisperse PEG solution. This graph is similar to the mass-spectrogram, previously obtained at the single molecule scale. $[37,46,56,58,59]$ As each peak matches with a precise chain length, we discriminate each component of a polydisperse mixture with a precision of one monomer (fig. 4(a)). As we did not use a monodisperse polymer, we do not know the exact number of monomeric unit. Nevertheless, according to these previous works, we can use a relative number of units. In figure 5(a), we observe that the blockade ratio $i_{b} / i_{0}$ decreases with the chain size, as it was previously observed with $\alpha$-Hemolysin channel. [56,59]

Now, we study the average dwell time distributions of all the blockades. First, we can plot a linear distribution, which is fitted with an exponential function $\exp \left(-t / T_{c}\right)$ where $T_{c}=830 \pm 10 \mu \mathrm{s}$ is a characteristic dwell time (fig. 4(c)). Another way is to plot a logarithmic distribution. Then, the fit function changes with a modified exponential function $t \times \exp \left(-t / T_{c}\right)$. [60]. This distribution is fitted
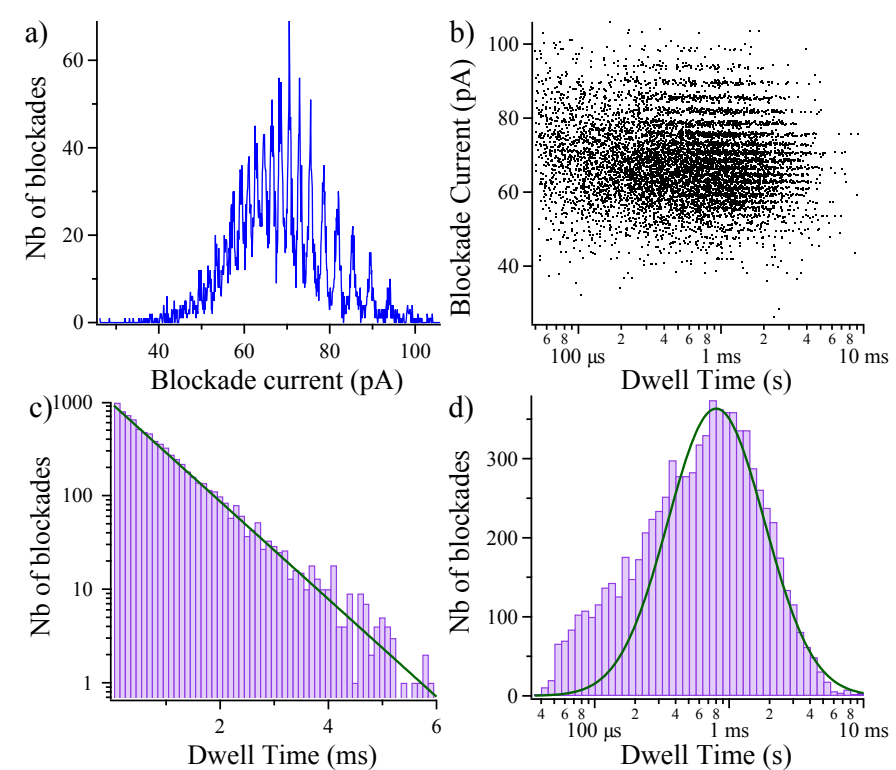

Fig. 4. Macromolecule transport : data analysis (a) Histogram of the blockade current $I_{b}$. The distribution is fitted with a Gaussian curve. (b) Scatter plot of the blockade current $I_{b}$ as a function of the corresponding dwell time $T_{t}$ of each blockade. (c) Linear distribution of the dwell time, fitted with an exponential function $\exp \left(-t / T_{c}\right)$. (d) Logarithmic distribution of the dwell time, fitted with a $\log$ normal function $\exp \left(-\left[\ln \left(t / T_{t}\right) / \sigma_{0}\right]^{2}\right)$. Aerolysin channel in presence of $10 \mu \mathrm{M}$ PEG1500 solution in a $3 \mathrm{M} \mathrm{KCl}$ buffer under a $-120 \mathrm{mV}$ applied potential.
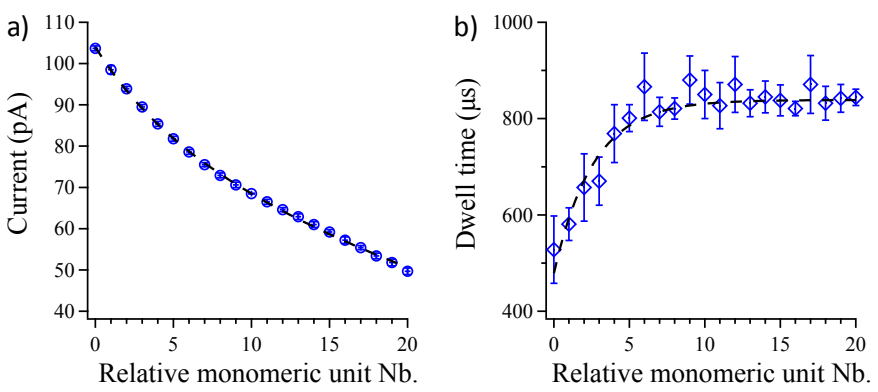

Fig. 5. Current blockade, dwell time vs relative number of monomeric unit. (a) Blockade current for each corresponding polymer chain discriminated in figure $4 \mathrm{a}$. The fit curve is a guide for the reader's eye (b) Dwell time obtained in the same way. Data are fit with an exponential function. Experiments are performed with $10 \mu \mathrm{M}$ PEG1500 in a $3 \mathrm{M} \mathrm{KCl}, 5 \mathrm{mM}$ Hepes, pH7.5 buffer at a $-120 \mathrm{mV}$ applied potential.

with a $\log$ normal function $\exp \left(-\left[\frac{\ln \left(t / T_{t}\right)}{\sigma}\right]^{2}\right)$ and is localized at the mean dwell time $T_{t}=800 \pm 50 \mu \mathrm{s}$ (fig. 4(d)). $T_{c}$ and $T_{t}$ have the same magnitude and are often correlated. [22] These distributions of all the polydisperse chains follow the same behavior as one monodisperse chain whereas we expect one time distribution for each polymer size.

Then, we consider each monodisperse population separately from the scatter plot (fig. 4(b)) to calculate each characteristic dwell time. The figure 5(b) shows that these dwell times increase exponentially with the number of 
monomeric units as it was previously observe with $\alpha$ Hemolysin or Aerolysin. [56,59] Nevertheless, they reach a steady state at $839 \pm 9 \mu$ s for higher relative monomeric unit numbers leading to an apparent dwell time plateau. This behavior could explain that we observe an apparent monodisperse distribution of dwell times (fig. 4(c)(d)). This limitation could be overcome by increasing salt concentration up to $4 \mathrm{M} \mathrm{KCl}$, [59] or increasing temperature. [37]

\section{Conclusion}

Conductance measurements through nanopores allow to study physical processes in confined phenomena. The detection of its variation is not so simple. Thus, some teams have developed specific algorithms to overcome experimental obstacles. In presence of a polymer chain, the conductance variation leads to the understanding of its interactions with the nanopore in terms of confinement or transport energies. [61] We could perform size discrimination of polydisperse PEG chains. We are able to detect each polymer chain according to their length from the current blockades measurements. Nevertheless, the sensitivity to dwell times in $3 \mathrm{M} \mathrm{KCl}$ must be enhanced by increasing salt concentration. Loïc Auvray showed us that the understanding of these fundamental processes is necessary to design and enhance new nanopore based devices as nanosensors or nanotools.

\section{Acknowledgments}

This research was supported by the "DEFI CNRS Instrumentation aux limites" 2016 \& 2017. We thank Nathalie Jarroux and Manuela Pastoriza-Gallego for their assistance. We thank Abdelghani Oukhaled for kindly providing ionic current traces through Aerolysin channel and for very instructive discussions. This publication is dedicated to Loïc Auvray's memory.

\section{Authors contributions}

All the authors were involved in the preparation of the manuscript. All the authors have read and approved the final manuscript.

L.B. and J.R. performed the data analysis, J.R., L.B., J.P., B.LP. prepared the manuscript.

\section{References}

1. S. Bhakdi, R. Fuessle, J. Tranum-Jensen, Proc. Natl. Acad. Sci. U. S. A. 78, 5475 (1981)

2. S.H. White, A.S. Ladokhin, S. Jayasinghe, K. Hristova, J. Biol. Chem. 276, 32395 (2001)

3. M.D. Peraro, F.G. van der Goot, Nat. Rev. Microbiol. 14, 77 (2015)
4. M. Todd, W. Longstaff, Journal of Petroleum Technology 24, 874 (1972)

5. J. Nittmann, G. Daccord, H.E. Stanley, Nature 314, 141 (1985)

6. F. Brochard, P.G. De Gennes, J. Chem. Phys. 67, 52 (1977)

7. M. Daoud, P. De Gennes, J. Phys. France 38, 85 (1977)

8. S. Daoudi, F. Brochard, Macromolecules 11, 751 (1978)

9. F. Brochard, P.G. De Gennes, J. Phys. Lett. 40, L399 (1979)

10. T. Sakaue, E. Raphaël, P.G. de Gennes, F. Brochard-Wyart, Europhys. Lett. 72, 83 (2005)

11. D. DeVault, J. Am. Chem. Soc. 65, 532 (1943)

12. C.S. Effenhauser, G.J.M. Bruin, A. Paulus, M. Ehrat, Anal. Chem. 69, 3451 (1997)

13. L. Beguin, B. Grassl, F. Brochard-Wyart, M. Rakib, H. Duval, Soft Matter 7, 96 (2011)

14. P.G. de Gennes, Proc. Natl. Acad. Sci. U. S. A. 96, 7262 (1999)

15. G. Guillot, L. Leger, F. Rondelez, Macromolecules 18, 2531 (1985)

16. J. Lal, Sunil K. Sinha, Loïc Auvray, J. Phys. II France 7, 1597 (1997)

17. E.P. Gilbert, L. Auvray, J. Lal, Macromolecules 34, 4942 (2001)

18. J.J. Kasianowicz, E. Brandin, D. Branton, D.W. Deamer, Proc. Natl. Acad. Sci. 93, 13770 (1996)

19. G. Oukhaled, L. Bacri, J. Mathe, J. Pelta, L. Auvray, Europhys. Lett. 82, 48003 (2008)

20. L. Brun, M. Pastoriza-Gallego, G. Oukhaled, J. Mathé, L. Bacri, L. Auvray, J. Pelta, Phys. Rev. Lett. 100, 158302 (2008)

21. B.J. Jeon, M. Muthukumar, ACS Macro Lett. 3, 911 (2014)

22. A. Meller, L. Nivon, E. Brandin, J. Golovchenko, D. Branton, Proc. Natl. Acad. Sci. U. S. A. 97, 1079 (2000)

23. A. Fennouri, C. Przybylski, M. Pastoriza-Gallego, L. Bacri, L. Auvray, R. Daniel, ACS Nano 6, 9672 (2012)

24. G. Oukhaled, J. Mathe, A.L. Biance, L. Bacri, J.M. Betton, D. Lairez, J. Pelta, L. Auvray, Phys. Rev. Lett. 98, 158101 (2007)

25. M. Pastoriza-Gallego, L. Rabah, G. Gibrat, B. Thiebot, F.G. van der Goot, L. Auvray, J.M. Betton, J. Pelta, J. Am. Chem. Soc. 133, 2923 (2011)

26. A. Oukhaled, L. Bacri, M. Pastoriza-Gallego, J.M. Betton, J. Pelta, ACS Chem. Biol. 7, 1935 (2012)

27. L. Bacri, A.G. Oukhaled, B. Schiedt, G. Patriarche, E. Bourhis, J. Gierak, J. Pelta, L. Auvray, J. Phys. Chem. B 115, 2890 (2011)

28. J. Ettedgui, J.J. Kasianowicz, A. Balijepalli, J Am Chem Soc 138, 7228 (2016)

29. D. Coglitore, A. Merenda, N. Giamblanco, L.F. Dumée, J.M. Janot, S. Balme, Phys. Chem. Chem. Phys. (2018)

30. M.T. Degiacomi, I. Iacovache, L. Pernot, M. Chami, M. Kudryashev, H. Stahlberg, F.G. van der Goot, M.D. Peraro, Nat Chem Biol 9, 623 (2013)

31. I. Iacovache, S. De Carlo, N. Cirauqui, M. Dal Peraro, F.G. van der Goot, B. Zuber, Nat. Commun. 7, 12062 (2016)

32. M.W. Parker, J.T. Buckley, J.P. Postma, A.D. Tucker, K. Leonard, F. Pattus, D. Tsernoglou, Nature 367, 292 (1994)

33. M. Muthukumar, J. Chem. Phys. 118, 5174 (2003)

34. M. Muthukumar, J. Chem. Phys. 132, 195101 (2010)

35. B. Cressiot, A. Oukhaled, G. Patriarche, M. PastorizaGallego, J.M. Betton, L. Auvray, M. Muthukumar, L. Bacri, J. Pelta, ACS Nano 6, 6236 (2012)

36. M. Moniatte, F. van der Goot, J. Buckley, F. Pattus, A. van Dorsselaer, FEBS Letters 384, 269 (1996)

37. F. Piguet, H. Ouldali, F. Discala, M.F. Breton, J.C. Behrends, J. Pelta, A. Oukhaled, Sci. Rep. 6, 38675 (2016) 
38. T. Gutsmann, T. Heimburg, U. Keyser, K.R. Mahendran, M. Winterhalter, Nat. Protoc. 10, 188 (2015)

39. M. Montal, P. Mueller, Proc. Natl. Acad. Sci. 69, 3561 (1972)

40. D. Deamer, M. Akeson, D. Branton, Nat. Biotechnol. 34, 518 (2016)

41. D. Pedone, M. Firnkes, U. Rant, Anal. Chem. 81, 9689 (2009)

42. A. Balijepalli, J. Ettedgui, A.T. Cornio, J.W.F. Robertson, K.P. Cheung, J.J. Kasianowicz, C. Vaz, ACS Nano 8, 1547 (2014)

43. Z. Gu, Y.L. Ying, C. Cao, P. He, Y.T. Long, Anal. Chem. 87, 907 (2015)

44. C. Plesa, C. Dekker, Nanotechnology 26, 084003 (2015)

45. J. Zhang, X. Liu, Y.L. Ying, Z. Gu, F.N. Meng, Y.T. Long, Nanoscale 9, 3458 (2017)

46. G. Baaken, N. Ankri, A.K. Schuler, J. Ruehe, J.C. Behrends, ACS Nano 5, 8080 (2011)

47. J.E. Hall, J Gen Physiol 66, 531 (1975)

48. J. Larkin, R.Y. Henley, M. Muthukumar, J.K. Rosenstein, M. Wanunu, Biophys. J. 106, 696 (2014)

49. C. Raillon, P. Granjon, M. Graf, L.J. Steinbock, A. Radenovic, Nanoscale 4, 4916 (2012)

50. F. Piguet, F. Discala, M.F. Breton, J. Pelta, L. Bacri, A. Oukhaled, The Journal of Physical Chemistry Letters 5, 4362 (2014)

51. J.H. Forstater, K. Briggs, J.W. Robertson, J. Ettedgui, O. Marie-Rose, C. Vaz, J.J. Kasianowicz, V. Tabard-Cossa, A. Balijepalli, Anal. Chem. 0, null (2016)

52. S.C. Liu, M.X. Li, M.Y. Li, Y.Q. Wang, Y.L. Ying, Y.J. Wan, Y.T. Long, Faraday Discuss. (2018)

53. S.E. Henrickson, M. Misakian, B. Robertson, J.J. Kasianowicz, Phys. Rev. Lett. 85, 3057 (2000)

54. B. Cressiot, E. Braselmann, A. Oukhaled, A.H. Elcock, J. Pelta, P.L. Clark, ACS Nano 9, 9050 (2015)

55. M.F. Breton, F. Discala, L. Bacri, D. Foster, J. Pelta, A. Oukhaled, The Journal of Physical Chemistry Letters 4, 2202 (2013)

56. J.E. Reiner, J.J. Kasianowicz, B.J. Nablo, J.W.F. Robertson, Proc. Natl. Acad. Sci. U. S. A. 107, 12080 (2010)

57. M. Boukhet, F. Piguet, H. Ouldali, M. Pastoriza-Gallego, J. Pelta, A. Oukhaled, Nanoscale 8, 18352 (2016)

58. J.W.F. Robertson, C.G. Rodrigues, V.M. Stanford, K.A. Rubinson, O.V. Krasilnikov, J.J. Kasianowicz, Proc. Natl. Acad. Sci. U.S.A. 104, 8207 (2007)

59. G. Baaken, I. Halimeh, L. Bacri, J. Pelta, A. Oukhaled, J.C. Behrends, ACS Nano 9, 6443 (2015)

60. O.V. Krasilnikov, C.G. Rodrigues, S.M. Bezrukov, Phys. Rev. Lett. 97, 18301 (2006)

61. A. Siria, M.L. Bocquet, L. Bocquet, Nature Reviews Chemistry 1, 0091 (2017) 